\title{
BAT X-RAY SURVEY. I. METHODOLOGY AND X-RAY IDENTIFICATION
}

\author{
M. Ajello, ${ }^{1}$ J. Greiner, ${ }^{1}$ G. Kanbach, ${ }^{1}$ A. Rau, ${ }^{2}$ A. W. Strong, ${ }^{1}$ and J. A. Kennea ${ }^{3}$ \\ Received 2007 July 18; accepted 2007 December 17
}

\begin{abstract}
We applied the maximum likelihood (ML) method, as an image reconstruction algorithm, to the BAT X-Ray Survey (BXS). This method was specifically designed to preserve the full statistical information in the data and to avoid mosaicking of many exposures with different pointing directions, thus reducing systematic errors when co-adding images. We reconstructed, in the 14-170 keV energy band, the image of a $90 \times 90 \mathrm{deg}^{2}$ sky region, centered on (R.A., decl.) $=\left(105^{\circ},-25^{\circ}\right)$, which BAT surveyed with an exposure time of $\sim 1 \mathrm{Ms}$ (in 2005 November). The best sensitivity in our image is $\sim 0.85$ mcrab or $2.0 \times 10^{-11} \mathrm{ergs} \mathrm{cm}^{-2}$. We detect 49 hard X-ray sources above the $4.5 \sigma$ level; of these, only 12 were previously known as hard X-ray sources (>15 keV). Swift XRT observations allowed us to firmly identify the counterparts for 15 objects, while 2 objects have Einstein IPC counterparts (Harris et al. 1990); in addition to those, we found a likely counterpart for 13 objects by correlating our sample with the ROSAT All-Sky Survey Bright Source Catalog (Voges et al. 1999). Seven objects remain unidentified. Analysis of the noise properties of our image shows that $\sim 75 \%$ of the area is surveyed to a flux limit of $\sim 1$ mcrab. This study shows that the coupling of the ML method to the most sensitive, all-sky surveying, hard X-ray instrument, BAT, is able to probe for the first time the hard X-ray sky to the millicrab flux level. The successful application of this method to BAT demonstrates that it could also be applied with advantage to similar instruments such as INTEGRAL IBIS.
\end{abstract}

Subject headings: galaxies: active — surveys — X-rays: binaries — X-rays: galaxies

Online material: machine-readable table

\section{INTRODUCTION}

More than 40 years after its discovery, the nature of the cosmic X-ray background (CXB) is still debated. Population synthesis models, based on unified active galactic nucleus (AGN) schemes, explain the CXB spectrum using a mixture of obscured and unobscured AGNs (e.g., Comastri et al. 1995; Gilli et al. 2001). According to these models, most AGN spectra are heavily absorbed, and about $85 \%$ of the radiation produced by super massive black hole accretion is obscured by dust and gas (Fabian \& Iwasawa 1999).

Deep soft X-ray surveys $(0.5-2.0 \mathrm{keV})$ were able to resolve the majority $(\approx 80 \%)$ of the CXB flux into discrete sources (Hasinger et al. 1998). However, the resolved fraction decreases with energy, being $\sim 50 \%-60 \%$ in the $6-8 \mathrm{keV}$ band (Giacconi et al. 2002; Rosati et al. 2002) and even less at $>8 \mathrm{keV}$; the missing CXB component has a spectral shape that is consistent with a population of as yet undetected, highly obscured AGNs (see Worsley et al. 2005).

It is important to realize that highly obscured objects are detectable in X-rays only above $10 \mathrm{keV}$. Moreover, most of the energy of the CXB is emitted around $30 \mathrm{keV}$ (Marshall et al. 1980), and the exact nature of the source population responsible for the background at these energies is unknown, primarily because of the low sensitivity of previous X-ray telescopes operating above $15 \mathrm{keV}$.

All these reasons together motivate more sensitive observations of the hard X-ray sky.

The Burst Alert Telescope (BAT; Barthelmy et al. 2005), on board the Swift mission (Gehrels et al. 2004), launched by NASA

\footnotetext{
1 Max-Planck-Institut für Extraterrestrische Physik, Postfach 1312, 85741 Garching, Germany.

2 Caltech Optical Observatories, Mail Stop 105-24, California Institute of Technology, Pasadena, CA 91125.

3 Department of Astronomy and Astrophysics, 525 Davey Laboratory, Pennsylvania State University, University Park, PA 16802.
}

on 2004 November 20, represents a major improvement in sensitivity for imaging of the hard X-ray sky. BAT is a coded mask telescope with a wide field-of-view (FOV, $120^{\circ} \times 90^{\circ}$ partially coded) aperture sensitive in the $15-200 \mathrm{keV}$ domain. BAT's main purpose is to locate gamma-ray bursts (GRBs). While chasing new GRBs, BAT surveys the hard X-ray sky with an unprecedented sensitivity. Thanks to its wide FOV and its pointing strategy, BAT monitors continuously up to $80 \%$ of the sky every day. Early results from the BAT survey (Markwardt et al. 2005) show that BAT is already 10 times more sensitive than the previous hard X-ray all-sky survey performed by HEAO-1 (Levine et al. 1984).

Coded mask telescopes are, until the advent of next generation hard X-ray focusing optics, among the most sensitive instruments able to image the sky in the hard X-ray domain. Objects in the FOV cast part of the mask pattern onto the detector plane. Since the sources' signal is coded by the mask onto the plane, this phase is also referred to as coding phase. Thus, a decoding procedure is required in order to reconstruct the original sky image. A variety of methods can be used to reconstruct the sky image in the case of a coded mask aperture (see Skinner et al. 1987 for a general discussion on reconstruction methods). Among them, standard cross-correlation of the shadowgram with a deconvolution array, the mask pattern, via fast Fourier transforms (FFTs), is the most often used. Generally, sky images are obtained for each individual observation, where an observation is defined as a period during which the attitude is stable and constant. Subsequently, another procedure, such as resampling and reprojecting, is needed in order to assemble the final allsky image.

Most of the extragalactic sources are very faint in the hard $\mathrm{X}$-ray band. Thus, their detection is challenging and requires sensitive techniques. We here describe the application of an alternative method which was designed to improve sensitivity, avoiding some of the disadvantages of the standard mask unfolding technique. 
This study has been performed in the framework of a campaign for optical spectroscopy analysis of a sample of "hard X-ray-selected" extragalactic sources aimed at identifying new Seyfert 2 galaxies. This paper discusses the method used to reconstruct the survey image and presents the source catalog. A second paper (Rau et al. 2007) describes in detail the optical campaign and the source identification process; the spectral analysis and the statistical properties of the source sample are discussed in Ajello et al. (2008).

The structure of the paper is as follows. In $\S 2$ we present details of the maximum likelihood (ML) method that was developed to analyze the BAT data. In $\S 3$ we describe the steps in the analysis we performed, and we present and discuss the results of our image reconstruction algorithm. The last section summarizes the results.

\section{SPATIAL MODEL FITTING}

We apply spatial model fitting, as described in Strong et al. (2005), to directly reconstruct the survey image from the raw detector data. Spatial model fitting means that a number of sky distributions, whose linear combination constitutes the model, are forward-folded through the full instrumental response in order to generate a model shadowgram. The model shadowgram, which is a linear combination of all model components, is then fitted in the full data space in order to get the most probable sky distribution. The actual search for unknown sources is then realized by moving a source probe in a grid over the sky. It is worth noting that no other steps, such as image mosaicking, are required at the end of this process. This method was successfully applied to different kinds of experiments (i.e., COMPTEL and INTEGRAL SPI; Diehl et al. 1995; Strong et al. 2005). Its development was driven by potential for reducing systematic errors and noise, e.g., the noise related to individual short images and the systematics when co-adding noisy images in the mosaicking procedure. This approach leads to an improvement in sensitivity over other methods, in particular reducing systematic errors from background variations and resampling. The information in the data is fully preserved and correctly treated in a statistical sense.

The likelihood is the probability of the observed BAT data given the model. For our case it is defined as the product of the probability for each detector of each observation:

$$
L=\prod_{i j k} p_{i j k},
$$

where

$$
p_{i j k}=\frac{\theta_{i j k}^{n_{i j k}} e^{-\theta_{i j k}}}{n_{i j k} !}
$$

is the Poisson probability of observing $n_{i j k}$ counts in pixel $i j$, during the $k$ th observation, when the number of counts predicted by the model is $\theta_{i j k}$.

The model is a linear combination of components; in the simplest case of one nonvariable source and one background component for each observation, we get

$$
\theta_{i j k}=c_{0}\left(A \otimes S^{\alpha_{0}, \delta_{0}}\right)_{i j k}+c_{k} B_{i j k},
$$

where $\left(A \otimes S^{\alpha_{0}, \delta_{0}}\right)$ is the convolution of the detector response $(A)$ and a source of unit flux $(S)$ at the sky position $\alpha_{0}, \delta_{0}$, and thus $\left(A \otimes S^{\alpha_{0}, \delta_{0}}\right)_{i j k}$ yields the prediction of counts from a unit flux source at the sky position $\alpha_{0}, \delta_{0}$ in detector $i j$, during observation $k ; B_{i j k}$ is the background prediction for pixel $i j$ in obser- vation $k$ and $c_{0}$ and $c_{k}$-values are the parameters we want to estimate.

For the analysis described in this paper, the background model comprises, for each observation, an empirical model (i.e., a twodimensional quadratic function similar to the one used by the tool batclean, as described in $\S 3.1$ ) and the model shadowgrams for all bright sources (see also $\S 3.1$ ). The actual fit to the background is performed only once; during the source search only the normalization of the background in each pointing is allowed to vary. Since sources detected at this stage are faint, the background normalizations are expected to vary by very small quantities. Indeed, we verified that such variations were less than $10^{-3}$ with respect to the background parameters determined before the source search.

In the future our method will make it possible to test more complex and physical background models (e.g., diffuse emissions).

\subsection{Parameter Model Estimation}

The parameter values are found by maximizing the likelihood function, or, equivalently, by maximizing its logarithm:

$$
\frac{\partial \ln L}{\partial \Lambda_{i}}=\frac{\partial}{\partial \Lambda_{i}}\left[\sum_{i j k} n_{i j k} \ln \left(\theta_{i j k}\right)-\sum_{i j k} \theta_{i j k}\right],
$$

where $\Lambda$ is the vector of the parameters. This translates into the following set of equations:

$$
\begin{gathered}
\frac{\partial \ln L}{\partial c_{0}}=\sum_{i j k}(A \otimes S)_{i j k}\left(\frac{n_{i j k}}{\theta_{i j k}}-1\right), \\
\frac{\partial \ln L}{\partial c_{k}}=\sum_{i j k} B_{i j k}\left(\frac{n_{i j k}}{\theta_{i j k}}-1\right), \forall k,
\end{gathered}
$$

which makes it possible to estimate all parameters simultaneously. This set of equations can be solved only numerically, and we use a modified Newton algorithm in order to find the solution.

\subsection{Source Significance}

In the case of a single source component, the source significance can be estimated using the likelihood-ratio test. For this application, the null hypothesis is that no point source exists at the position under consideration and the background model can explain all the data. The alternative hypothesis is the converse. Two maximizations have to be done in order to calculate the likelihood $L_{0}$ of the background (null hypothesis) and the likelihood of both source and background for the alternative hypothesis $L_{1}$. The test statistic

$$
T_{s} \equiv-2\left(\ln L_{0}-\ln L_{1}\right)
$$

is expected, from Wilks's theorem (Wilks 1938), to be asymptotically distributed as $\chi_{n}^{2}$ in the null hypothesis, where $n$ is the additional number of free parameters that are optimized for the alternative hypothesis. Since in our case the source intensity is the only additional free parameter, the test statistic is expected to follow the $\chi_{1}^{2}$ distribution. Thus, the significance of a detection can be addressed as

$$
S=\int_{T_{s}}^{\infty} \frac{1}{2} \chi_{1}^{2}(x) d x
$$


which, after changing variables, becomes

$$
S=\int_{\sqrt{T_{s}}}^{\infty} \frac{e^{-y^{2} / 2}}{(2 \pi)^{1 / 2}} d y
$$

Equation (9) is exactly the integral of the standard normal distribution from $T_{s}^{1 / 2}$ to $\infty$, and so the significance of the detection is

$$
T_{s}^{1 / 2} \sigma=\sqrt{-2\left(\ln L_{0}-\ln L_{1}\right)} \sigma=n \sigma .
$$

Hence, by definition, and if everything is done correctly, the significance fluctuations must be distributed as a normal Gaussian.

\subsection{Method Implementation}

In the case of large detector counts, the likelihood maximization is equivalent to the $\chi^{2}$ minimization, with the $\chi^{2}$ problem having the advantage that it can be solved faster analytically. We have verified that in the case of large detector counts $(\geq 20)$ and large numbers of observations $(\geq 100)$ the two solutions are very similar, and from now on we use the $\chi^{2}$ solution.

The algorithm used is a parallelized implementation of spidiffit (Strong et al. 2005) used for INTEGRAL SPI data analysis. Parallelization was needed because of the large size of the problem we are dealing with. The typical execution time needed to compute the analytical $\chi^{2}$ solution scales with $n^{2}$, where $n$ is the number of data points to fit (i.e., number of BAT detectors, 32,768, multiplied by the number of observations). A single minimization with 2600 observations takes nearly $90 \mathrm{~s}$; the total execution time to generate a map of $450 \times 450$ pixels would be $\sim 200$ days. This time has been reduced to $<15$ days using an average of 15 CPUs. We remark also that it is the first time that such an approach has applied to a problem of this large size.

As shown in equation (3), the model is a linear combination of different components which can be specified at the input of the program. Source and background components are in general treated in different ways. Sources are assigned a single free parameter (their average intensity), while, as already discussed, the background components are allowed to vary from pointing to pointing. However, in case of variable sources, the user can specify that the source intensity is left as a free parameter in all pointings (or in time-contiguous groups of them).

We remark that for the analysis presented in the next sections, the program has been used in its simplest configuration, with only one constant source and the normalizations of the per-pointing backgrounds allowed to vary. However, after the source search had been performed and source candidates identified we used the ability to simultaneously fit all sources (each of which was again assumed constant in time). In fact, the simultaneous fit of all sources yields the best parameters (significances and fluxes) and allows us to discard spurious detections. When the analysis is based on a large number of observations, correlation (cross-talk) between sources is negligible.

\subsection{Instrumental Response}

As shown in equation (3), the first part of the model represents the source component (or components if more than one), and this is given in the most simple form by a pointlike source at position $\alpha_{0}, \delta_{0}$ in the sky, forward-folded with the instrumental

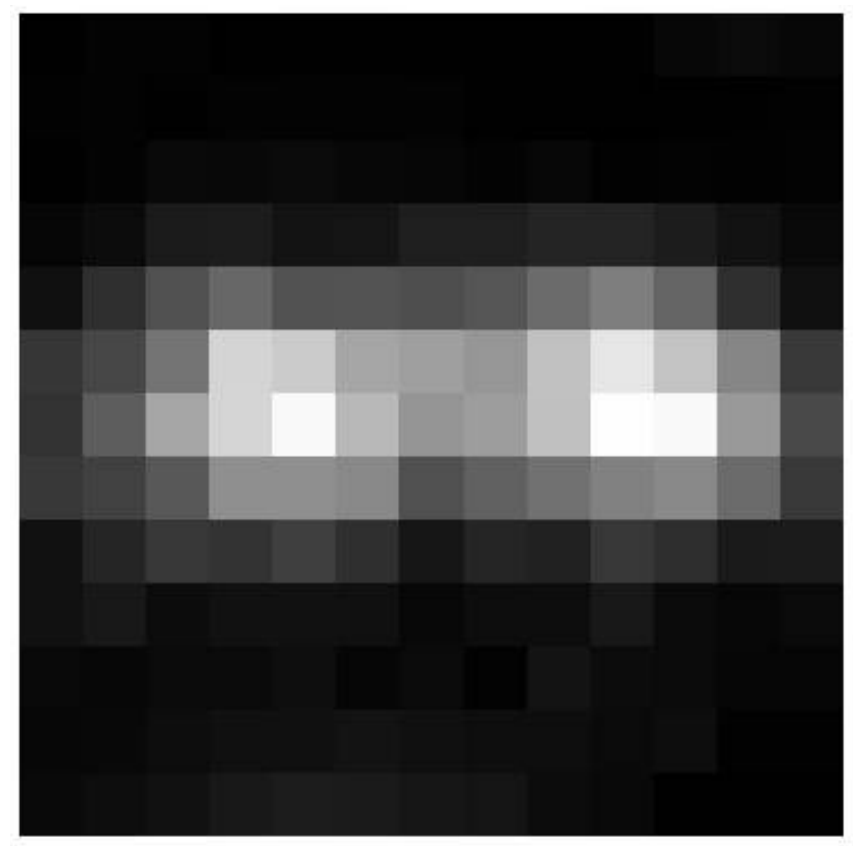

FIG. 1.- Image of LMC X-1 (right) and PSR B0540-69.3 (left) clearly separated. The pixel size is $5^{\prime}$ and the map is about $1 \times 1 \mathrm{deg}^{2}$.

response. We have used a large set of Crab observations $(>1000)$ to develop a parameterized diagonal full instrumental response which enables us to predict the expected counts (essentially the term $A \otimes S^{\alpha_{0}, \delta_{0}}$ of eq. [3]) from a unit flux source as a function of energy and position in the field of view. The parameterized instrumental response was obtained in the following way (standard BAT software is reported in parentheses):

1. for each Crab observation a model shadowgram for the source position is computed (tool batmaskwtimg);

2. for each Crab observation the source model and the standard background components of batclean (see $\S 3.1$ ) are fitted to the data;

3. the normalizations of the source components (in the different observations) are parameterized as a function of off-axis angle.

The parameterized instrumental response is thus, for a given source position, the product of the model shadowgram described in equation (1) multiplied by a coefficient computed from the parameterization derived in equation (3). In this way the instrumental response accounts for the off-axis ${ }^{4}$ variation of the detected source intensity which the batmaskwtimg model does not take into account. The response, derived in this way, agrees with measured values to within $1 \sigma$ anywhere in the FOV.

To improve the speed of the code during source search, the full instrumental response was precomputed over a $6^{\prime}$ pitch grid in the whole BAT FOV.

To conclude, in Figure 1 we show the imaging reconstruction capabilities of our approach; two close-by faint sources, LMC X-1 and PSR B0540-69.3, are clearly detected in the image obtained using $\sim 2600$ observations. The good angular resolution of BAT is also preserved by our imaging reconstruction algorithm; in fact the two sources are separated by just $25^{\prime}$ (for comparison the BAT point-spread function is $22^{\prime}$ ).

4 The reader can find more details about the off-axis variation of the source signal and other effects in the Appendix A.1 of Ajello et al. (2008). 


\section{ANALYSIS}

In this section we describe the application of the ML method to reconstruct the image of $\sim \frac{1}{8}$ of the sky using 8 months of BAT data.

\subsection{Data Selection and Screening}

We used 8 months of data, from 2005 April (when BAT data became public) to 2005 November. In order to secure optical follow-up with a dedicated observing campaign at La Silla, Chile, in 2006 January, we selected only observations with angular separation less than $45^{\circ}$ from the zenith (R.A. $=105^{\circ}$, decl. $=$ $\left.-25^{\circ}\right)$. The all-sky analysis, still within the capabilities of modern supercomputers, will be left to a future study.

Swift BAT survey data are in the form of 80 channels detector plane histograms (DPHs) with typical exposure time of $300 \mathrm{~s}$. In order to have a suitable clean data set as input of the imaging reconstruction algorithm described in $\S 2$, preprocessing must be carried out on the raw survey data. This preprocessing phase accomplishes two different goals: (1) data quality is monitored along the processing and (2) the very bright sources detected during each single observations are localized and inserted in the background model of the imaging reconstruction algorithm. The latter procedure can be justified as follows. The brightest sources (except the Crab Nebula) are known to be highly variable. However, since they are detected in general at high significance in a single observation, their intensities can be determined with good accuracy. Thus, inserting bright sources in the background model, rather than treating them as several independent components in the source model, makes it possible to handle source variability in a natural way without increasing the size of the problem.

All the preprocessing was carried out using the latest available version of the Swift software contained in the HEASOFT 6.0.3. Below we report in parentheses the name of the standard BAT tools used during our preprocessing.

For each DPH, our preprocessing pipeline, does the following operations:

1. Data are rebinned in energy channels according to the gainoffset map generated on board (baterebin).

2. The DPH is integrated along the energy axis, between 14 and $170 \mathrm{keV}$, and a detector plane image (DPI) is generated (batbinevt).

3. A detector quality mask is created, where hot and cold pixels are masked out (bathotpix). These pixels are identified as the wings of the distribution of counts for a given observation; in general $2 \%$ (and so roughly $1 \%$ on each side) of the distribution is excised.

4. An empirical background model is fitted to the DPI (batclean). ${ }^{5}$

5. The DPI and the background model are input to a FFT deconvolution algorithm which generates the sky image (batfftimage).

6. Source detection takes place on the sky image and a catalog of all sources detected with a signal-to-noise ratio $(\mathrm{S} / \mathrm{N})$ $>6 \sigma$ is created (batcelldetect).

7. A model for each detected source is created, and it is added to the background model of step 4 . The source model is created

\footnotetext{
5 The empirical background model built-in in batclean fits (for a given energy range) a quadratic spatial function plus a series of models which take care of detector edge effects for a total of 14 parameters. The user is also free to include sources or different background models. The reader can find more details about the batclean background model in the documentation included in the HEASOFT package (a copy of it is also available at http:// heasarc.nasa.gov/ lheasoft/ftools/headas/batclean.html).
}

TABLE 1

Fraction of Rejected Data due to Any Single Criterion

\begin{tabular}{|c|c|}
\hline Type & $\begin{array}{c}\text { Fraction } \\
(\%)\end{array}$ \\
\hline Star tracker lock.. & 9.7 \\
\hline Pointing stability..... & 17.4 \\
\hline Outside SAA............ & 10 \\
\hline Exposure $>200 \mathrm{~s}$ & 7 \\
\hline 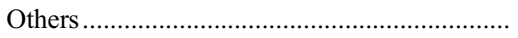 & $<1$ \\
\hline 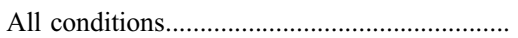 & 34 \\
\hline
\end{tabular}

using the measured source coordinates. These coordinates were preferred to the catalog position because of nontrivial systematic effects which produced a shift in the measured source coordinates as a function of position in the FOV. ${ }^{6}$

8. Steps 4 to 7 are repeated until no new sources are detected in a single $300 \mathrm{~s}$ observation.

In order to have the cleanest data set possible, we have applied cuts on the quality of the data. During the steps above data are screened on the basis of the following conditions:

1. lock of the star tracker and pointing stability;

2. spacecraft being outside of the South Atlantic Anomaly (SAA). This information is reported in the housekeeping data and is referred to a fiducial point inside the SAA;

3. BAT array rate $<18,000$ counts $\mathrm{s}^{-1}$;

4. exposure being larger than $200 \mathrm{~s}$;

5. reduced $\chi^{2}$ of the background fit $<1.5$;

6. $>9 \sigma$ detected sources must be within a distance of $0.1^{\circ}$ from a known source otherwise they are thought to be spurious or transient. The observation is flagged for a later analysis, but not inserted into the final data set.

In Table 1 we list the fraction of exposure which is rejected if a single data quality cut is applied to the data used for this analysis. For the current data set, $\sim 34 \%$ of the overall exposure time was rejected because the data did not meet one or more of the above-mentioned criteria.

After processing and screening the data according to these criteria, the final data set includes 2671 observations. These observations are input to the imaging reconstruction algorithm described in $\S 2$. Figure 2 shows the total exposure map of all pointings.

All sources detected during the preprocessing phase are listed in Table 2 along with their identification, their maximum and total significance (computed as the sum of the squared of significances) from this per-pointing analysis, and the number of detections. The distribution of the offsets of sources in Table 2 from their catalog counterpart is reported in Figure 3. The same graph shows the extremely good location accuracy of BAT, which locates $95 \%$ of all sources detected in single pointings within $2.2^{\prime}$ radius. In order to understand the dependence of location accuracy on the source significance, we have analyzed all per-pointing detections (see Fig. 4) and determined that the offset varies with significance according to

$$
\mathrm{OFFSET}=4.94 \pm 0.68 \times(\mathrm{S} / \mathrm{N})^{-0.59( \pm 0.05)}(\operatorname{arcmin}) .
$$

This analysis is based only on sources detected during individual pointings.

\footnotetext{
${ }^{6}$ See http://swift.gsfc.nasa.gov/docs/swift/analysis/bat_digest.html for more details.
} 


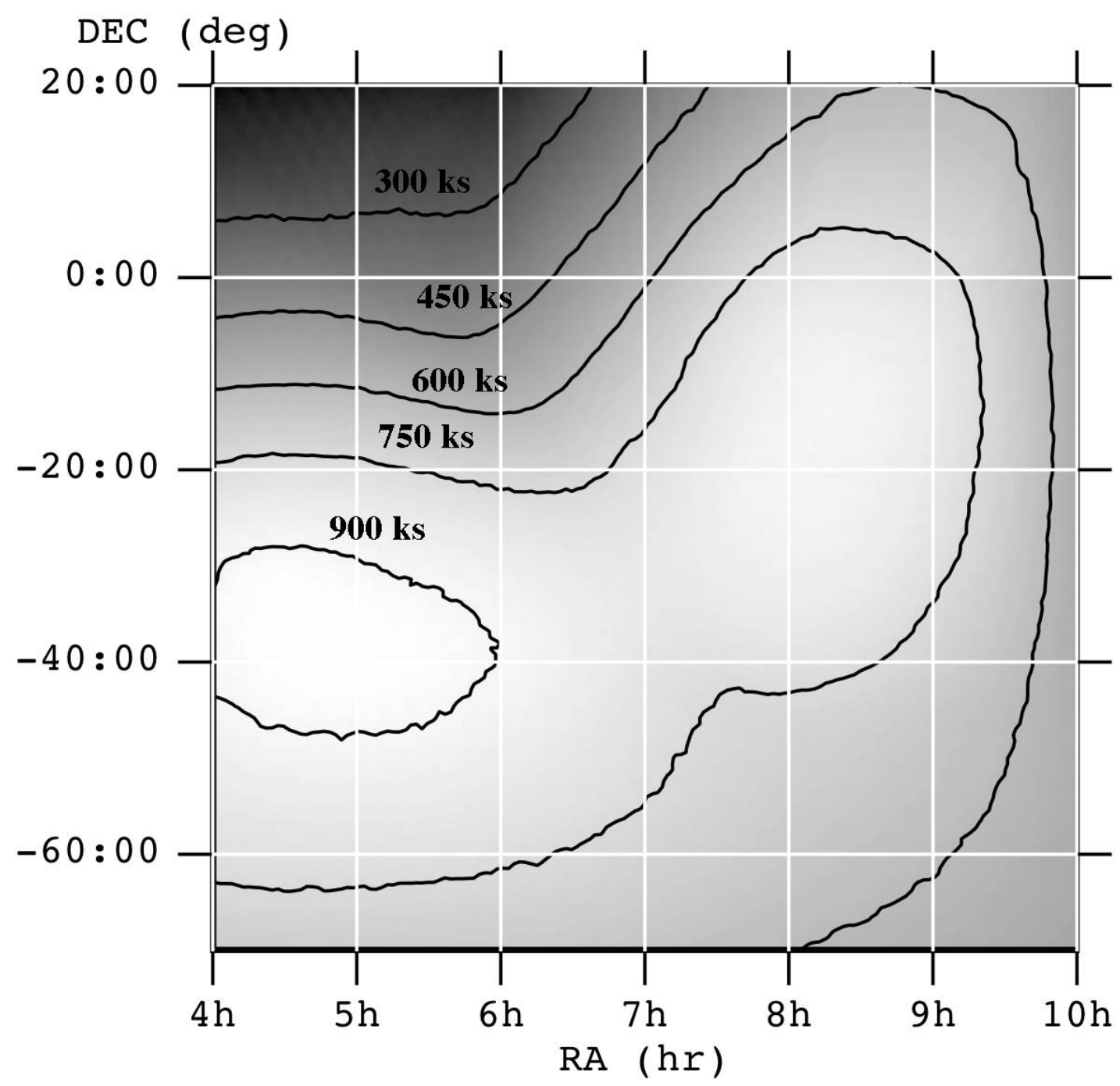

FIG. 2.- Exposure map (declination, degrees, on left axis; right ascension at bottom), corrected for telescope vignetting, of the survey field presented in text after data screening.

\subsection{Imaging Reconstruction}

The 2671 DPIs, along with their background models (created at step 4 in $\S 3.1$ ), are input of the imaging reconstruction algorithm. For this analysis we have used one parameter for the source component; moreover, we have allowed the normalization of the background component to vary separately in each pointing, leading to a total of 2672 parameters. The map is built in small segments of $5 \times 5 \mathrm{deg}^{2}$. A pixel size of $12^{\prime}$ was chosen as the best compromise between computational time and the resampling factor of the PSF ( $\sim 2$ in this case). The significance image is shown in Figure 5.

\subsection{Setting the Significance Threshold}

There are several approaches in order to derive the best significance threshold. The ML method leads to perfectly symmetric normal Gaussian noise in the pixels of the reconstructed image. Thus, the most straightforward approach is setting the threshold as the absolute value of the lowest negative fluctuation. In this case, since the negative fluctuations are given by noise, one should expect no false detection above this threshold.

As it can be seen in the significance distribution reported in Figure 6, no negative fluctuations larger than -4.3 are found.
Taking into consideration the number of trials and the normal Gaussian distribution, we concluded that above the threshold $\mathrm{S} / \mathrm{N}$ of 4.5 the expected number of false detections is 0.7 . We also made a Monte Carlo simulation generating a large number $(>1000)$ of sky images with Gaussian noise. We then counted all the excesses above the $4.5 \sigma$ level and found out that the number of expected false detection is 1.01, in agreement with the previous finding. A contamination of our sample of sources by $\sim 1$ spurious detection was judged to be a good compromise between detection sensitivity and sample corruption (see $\S 3.5$ for the chance connected to have a higher contamination). Hence, we decided to fix the threshold to $4.5 \sigma$.

\subsection{Noise Properties and Sky Coverage}

The sky coverage, for a given survey, is the distribution of the survey's area as a function of limiting flux. Knowledge about the sky coverage is particularly important when computing the number-flux relation (also known as $\log N-\log S$ distribution). We leave the derivation of the number-flux relation to a separate paper (Ajello et al. 2008), but we are interested in deriving the sky coverage here, as it brings crucial information about the sensitivity and noise properties of the survey. 
TABLE 2

Sources Detected during Data Screening

\begin{tabular}{|c|c|c|c|c|c|c|}
\hline $\begin{array}{l}\text { R.A. (J2000.0) } \\
\quad(\operatorname{deg})\end{array}$ & $\begin{array}{c}\text { Decl. (J2000.0) } \\
(\mathrm{deg})\end{array}$ & $\begin{array}{l}\mathrm{S} / \mathrm{N} \\
(\max )\end{array}$ & Total S/N & Number of Detections & Type & ID \\
\hline $19.1746 \ldots \ldots \ldots \ldots \ldots \ldots$ & -73.4559 & 16.9 & 47.7 & 22 & HXB & SMC X-1 \\
\hline $58.8665 \ldots \ldots \ldots \ldots \ldots \ldots \ldots \ldots$ & +31.0270 & 8.3 & 8.3 & 1 & HXB & $\mathrm{V}^{*} \mathrm{X}$ Per \\
\hline $83.2654 \ldots \ldots \ldots \ldots \ldots \ldots$ & -66.3567 & 11.5 & 35.0 & 17 & HXB & LMC X-4 \\
\hline $83.6265 \ldots \ldots \ldots \ldots \ldots$ & +22.0079 & 126.9 & 1505 & 581 & PSR & Crab \\
\hline $84.7121 \ldots \ldots \ldots \ldots \ldots \ldots$ & +26.2949 & 90.5 & 195 & 61 & HXB & 1A $0535+26$ \\
\hline $94.2964 \ldots \ldots \ldots \ldots \ldots \ldots . .$. & +9.1199 & 8.7 & 12.6 & 3 & LXB & $\mathrm{V}^{*} \mathrm{~V} 1055$ Ori \\
\hline $117.2030 \ldots \ldots \ldots \ldots \ldots \ldots$ & -67.7483 & 8.0 & 30.0 & 15 & LXB & EXO 0748-676 \\
\hline $135.5234 \ldots \ldots \ldots \ldots \ldots \ldots$ & -40.5565 & 139.3 & 917 & 888 & HXB & Vel X-1 \\
\hline $152.4996 \ldots \ldots \ldots \ldots \ldots \ldots \ldots$ & -58.2222 & 13.7 & 38.8 & 18 & HXB & GRO J1008-57 \\
\hline $170.2396 \ldots \ldots \ldots \ldots \ldots \ldots$ & -60.5556 & 50.2 & 161.7 & 79 & HXB & Cen X-3 \\
\hline $176.9183 \ldots \ldots \ldots \ldots \ldots$ & -61.9794 & 8.9 & 8.9 & 1 & HXB & $\mathrm{V}^{*}$ V830 Cen \\
\hline $186.6980 \ldots \ldots \ldots \ldots \ldots \ldots$ & -62.7649 & 98.0 & 401 & 284 & HXB & GX 301-2 \\
\hline $201.4286 \ldots \ldots \ldots \ldots \ldots \ldots$ & -43.0056 & 8.8 & 12.6 & 2 & Sy2 & Cen A \\
\hline $235.5705 \ldots \ldots \ldots \ldots \ldots \ldots$ & -52.3704 & 12.2 & 14.4 & 2 & HXB & $\mathrm{V}^{*} \mathrm{QV}$ Nor \\
\hline $243.0916 \ldots \ldots \ldots \ldots \ldots \ldots$ & -52.4028 & 16.1 & 40.7 & 19 & LXB & H $1608-522$ \\
\hline $244.9860 \ldots \ldots \ldots \ldots \ldots$ & -15.6526 & 25.5 & 76.0 & 24 & LXB & Sco X-1 \\
\hline $247.9922 \ldots \ldots \ldots \ldots \ldots \ldots$ & -48.8173 & 8.8 & 11.5 & 2 & HXB & IGR J16318-4848 \\
\hline $250.2720 \ldots \ldots \ldots \ldots \ldots \ldots$ & -53.7497 & 8.9 & 19.6 & 6 & LXB & H 1636-536 \\
\hline $251.4481 \ldots \ldots \ldots \ldots \ldots$ & -45.6088 & 13.1 & 21.8 & 6 & LXB & GX $340+0$ \\
\hline $255.1860 \ldots \ldots \ldots \ldots \ldots \ldots$ & -41.6435 & 27.0 & 38.9 & 8 & HXB & EXO 1657-419 \\
\hline $255.9813 \ldots \ldots \ldots \ldots \ldots \ldots$ & -37.8334 & 50.2 & 98.3 & 22 & HXB & $\mathrm{V}^{*}$ V884 Sco \\
\hline $256.4394 \ldots \ldots \ldots \ldots \ldots$ & -36.4345 & 12.1 & 13.2 & 2 & LXB & Sco X-2 \\
\hline
\end{tabular}

Notes.-Note that the significance of the detection depends on the source intensity, exposure, and on the position in the FOV. The total $\mathrm{S} / \mathrm{N}$ was computed as the sum of the squared significances in each observation.

The sky coverage as a function of the minimum detectable flux $F_{\min }$ is defined as the sum of the area covered to fluxes $f_{i}<F_{\min }$ :

$$
\Omega\left(<F_{\min }\right)=\sum_{i}^{N} A_{i}, \quad f_{i}<F_{\min },
$$

where $N$ is the number of image pixels and $A_{i}$ is the area associated with each of them.

We have followed two procedures to compute the sky coverage of our survey area:

1. The ML method produces a flux map and an error map as output of the fitting procedure. In order to get the sky coverage

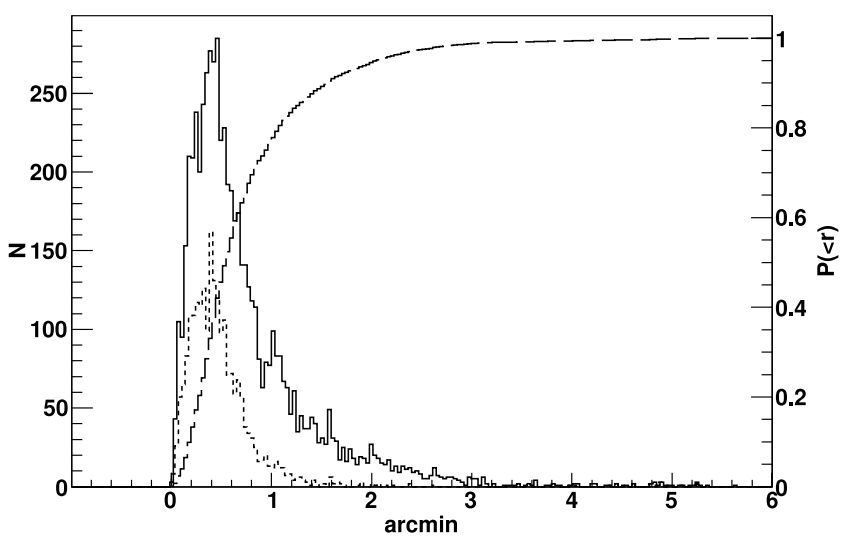

FIG. 3.- The solid line is the distribution of the offsets of sources detected in the individual observations (see Table 2) from their catalog position. The inner histogram (dotted line) shows the detections of the Crab, while the dashed line is the cumulative distribution of all detections. The $90 \%$ and $95 \%$ confidence limits are at radii of $1.8^{\prime}$ and $2.2^{\prime}$, respectively. we multiplied the error map by the $4.5 \sigma$ threshold $\mathrm{S} / \mathrm{N}$ and then counted the area as in equation (12).

2 . We computed the local (flux) image variance using a sliding annular region whose internal and external radii were 5 and 30 pixels, respectively. The noise of a given pixel is thus computed as the variance of the pixels contained in the annulus centered on it. The central pixels are excised so that the background does not include contamination from the source region. This map is a true representation of the noise in our image. Again, we multiplied this noise map by our detection threshold of $4.5 \sigma$ and then counted the area as in equation (12).

No significant difference appear between the sky coverage computed in these two ways, suggesting that the error computed by the ML method is very close to (if not the same as) the

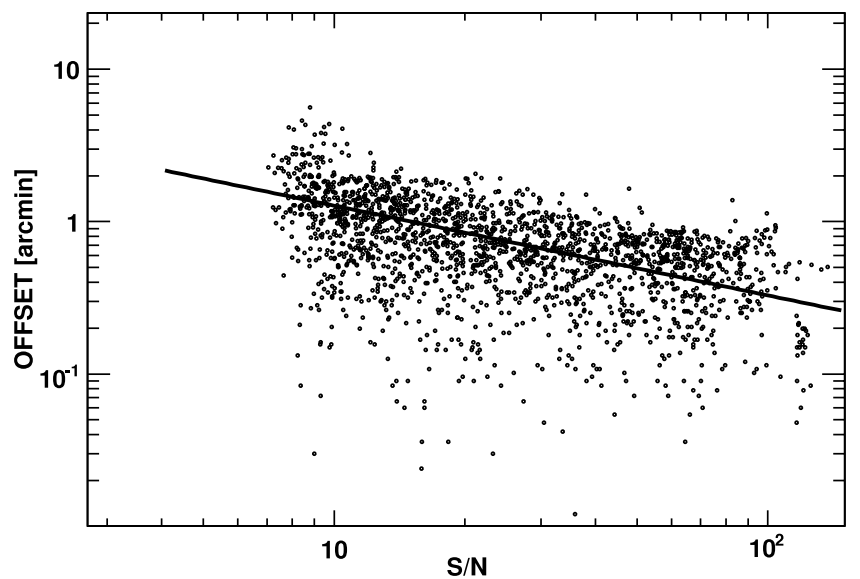

FIG. 4.-Offset from catalog position of sources detected in individual observations as a function of $\mathrm{S} / \mathrm{N}$. The solid line is the function described in eq. (11). 


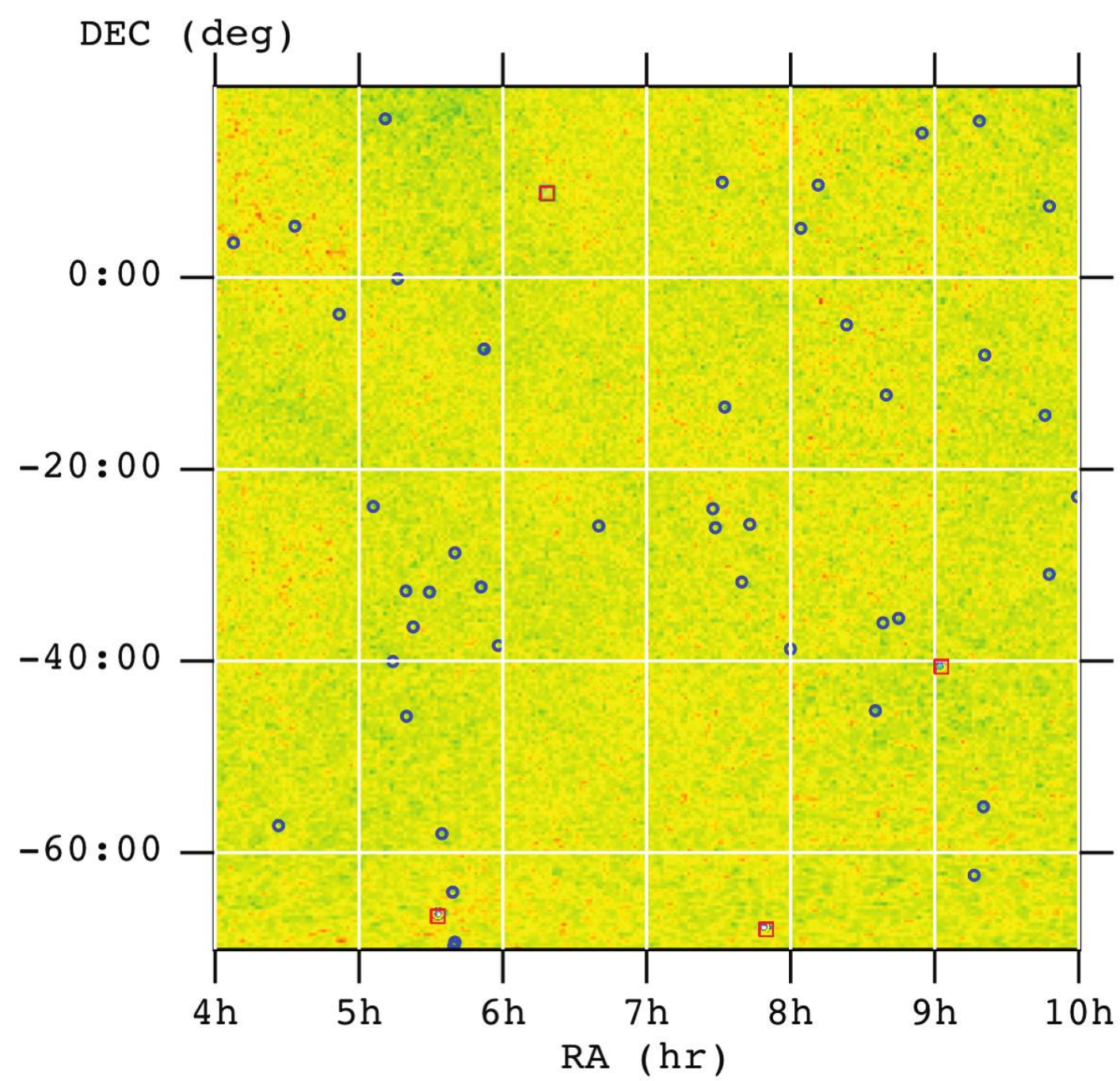

Fig. 5. - Significance map of the $90 \times 90 \mathrm{deg}^{2}$ surveyed area (declination, in degrees, on left axis; right ascension at bottom). Blue circles mark all sources above $4.5 \sigma$ presented in Table 4, while red squares show the position of bright sources which were included in the background model.

real noise term of the sky image. In the left panel of Figure 7 we report the sky coverage of the entire area and for the extragalactic portion of the sky (selected imposing $|b|>15^{\circ}$ ). As can be seen from the sky coverage, $>75 \%$ of the surveyed area is sensitive to fluxes $\sim 1 \mathrm{mcrab}$, and all of it to fluxes $>2.0$ mcrab.

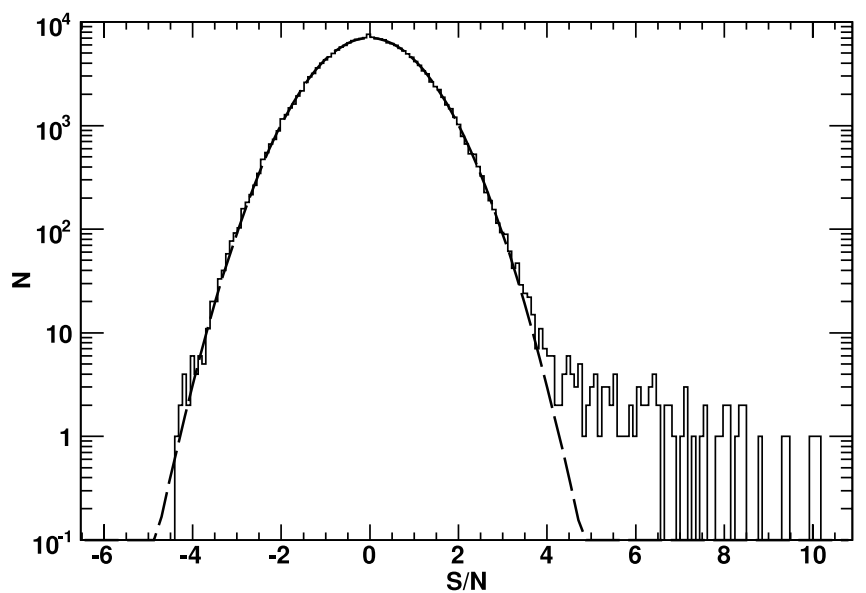

FIG. 6.- $\mathrm{S} / \mathrm{N}$ distribution. The dashed line is an overlaid Gaussian with $\sigma=1$.
The limiting sensitivity in our image is a bit less than 0.9 mcrab (or $2.05 \times 10^{-11}$ ergs $\mathrm{cm}^{-2} \mathrm{~s}^{-1}$ ).

The analysis of the pixel noise as a function of exposure time (reported in the right panel of Fig. 7) shows that the survey sensitivity scales $\propto T^{-0.5}$, denoting that systematic errors do not dominate over statistical ones. We then compared our survey sensitivity to recent results from the BAT and INTEGRAL ISGRI hard X-ray surveys (Markwardt et al. 2005; Bassani et al. 2006). In order to perform the comparison, we transformed the sensitivities provided by the authors in different bands to sensitivities in a common band (20-100 keV); the comparison, which is shown in Table 3, is done in two ways: once by taking into account the threshold $\mathrm{S} / \mathrm{N}$ used by the authors in their work, and then once again based on a common $5 \sigma$ equivalent sensitivity. The main result is that for $1 \mathrm{Ms}$ of exposure, our survey is one of the most sensitive.

\subsection{Source Detections and Fluxes}

Source detection on the reconstructed image is a straightforward process, since significance and flux maps are direct results of our reconstruction algorithm. All noncontiguous pixels which meet the criterion $\mathrm{S} / \mathrm{N}>\mathrm{S} / \mathrm{N}_{\text {threshold }}$ are identified. However, at this stage we have lowered our detection threshold to 

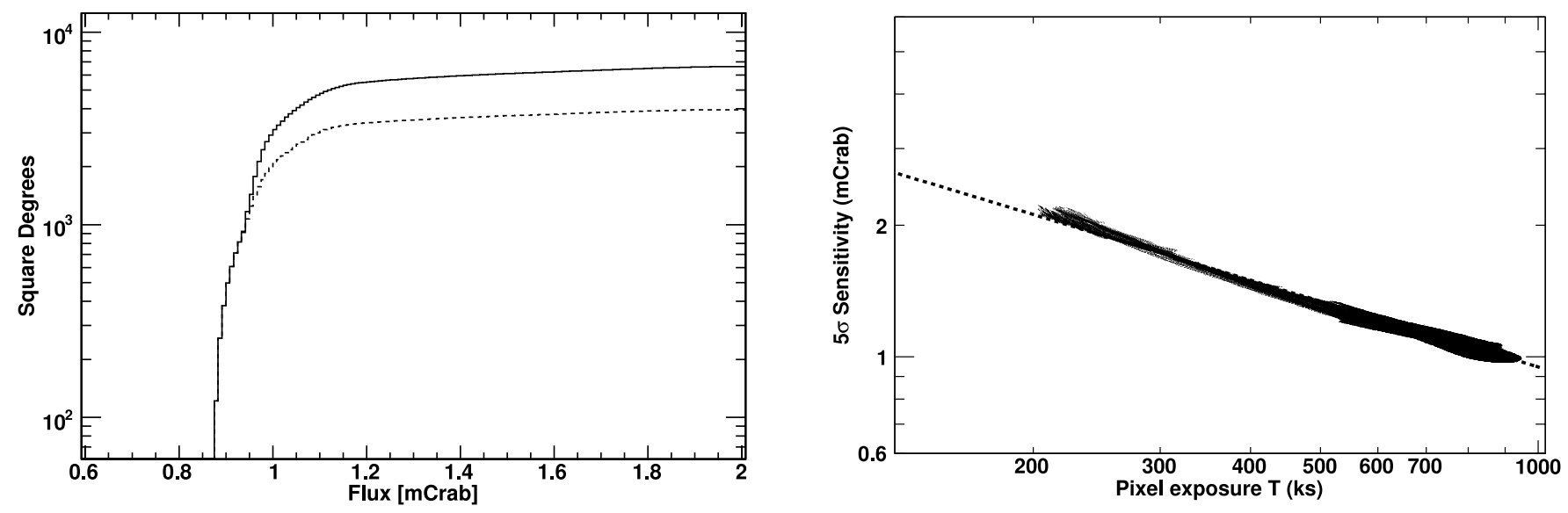

Fig. 7. - Left: Sky coverage as a function of minimum detectable flux for $\mathrm{S} / \mathrm{N}=4.5 \mathrm{in}$ the $14-170 \mathrm{keV}$ band. As can be seen, $75 \%$ of the surveyed area is covered to $1 \mathrm{mcrab}$. The dashed line is the sky coverage for $|b|>15^{\circ}$. Right: Pixel $5 \sigma$ sensitivity threshold as a function of pixel exposure time. The dashed line is the fit to data points corresponding to the function $3.0 \mathrm{mcrab}(T / 100 \mathrm{ks})^{-0.5}$.

(an optimally chosen) $3.5 \sigma$. Indeed, our procedure of using a precomputed response over a $6^{\prime}$ pitch grid on a $12^{\prime}$ pixel-size map might produce a small loss in the reconstructed sources' fluxes and significances. In order to overcome this problem, we have generated, for all the above candidates, a $5^{\prime}$ pitch grid map using the correct instrumental response (i.e., not precomputed on a $6^{\prime}$ pitch grid). One such map has already been shown in Figure 1. Only those candidates whose significance, as derived from the oversampled small maps, exceeds the $4.5 \sigma$ threshold are kept in the sample and fitted with the instrumental pointspread function (the batcelldetect is used here) in order to determine the most accurate source parameters. This procedure allows us to recover the correct source significance and flux at the cost of a slightly larger number of false detections. Indeed, due to the increased number of trials, the expected number of false detection is now 1.5 . We remark that our map is one realization over many; thus there exists a nonzero probability that the number of false detection exceeds the (averaged) value estimated here. Our Monte Carlo simulation shows that the probability of getting a number of false detections of 2,3 , and 4 is, respectively, $0.21,0.09$, and 0.02 .

\subsection{Detected Sources}

We have detected 49 hard X-ray sources in our survey. Four of these sources are residuals caused by imperfect modeling (and inclusion in the background model) of bright sources which are detected in individual DPHs. These four sources (LMC X-4, EXO 0748-676, Vel X-1, and $\mathrm{V}^{*} \mathrm{~V} 1055$ Ori) are still detected in the reconstructed image with a $\mathrm{S} / \mathrm{N}$ of $20-40$.
In Table 4 we report the coordinates and fluxes of all 45 serendipitous objects detected above the $4.5 \sigma$ detection threshold.

We have correlated our sources with the ROSAT All-Sky Survey Bright Source Catalogue (Voges et al. 1999) in the same way as in Stephen et al. (2006). In Figure 8 we report the number of BAT sources which have at least one ROSAT source within a given radius. Also, to understand the contribution of chance coincidences to these associations, we performed a Monte Carlo simulation using $5 \times 10^{5}$ positions randomly distributed in our field. Due to nonuniformity in the distribution of ROSAT sources, the probability of a chance association increases slightly toward negative Galactic latitudes. Taking into account the highest density of ROSAT sources (for $-40^{\circ}<b<-20^{\circ}$ ), we find from Figure 8 that using a radius of $300^{\prime \prime}$ for the identification of our sources will yield a probability of chance coincidence of 0.015 (one wrong identification overall).

The same figure also yields information about the BAT pointspread function location accuracy (PSLA), as the BAT uncertainty in the position dominates the ROSAT error. Thus, assuming that the ROSAT position is the "true" source position and considering only the ROSAT associations, we fitted an inverted Gaussian to the curve of Figure 8 (see Fig. 9); we derived that $95 \%$ and $99 \%$ of all spatial coincidences are within $3.3^{\prime}$ and $5^{\prime}$, respectively. Thus, using a $5^{\prime}$ radius for source identification yields the best compromise between probability of finding the BAT counterpart and chance coincidence.

It is not surprising that $<70 \%$ of our sample is correlated with the ROSAT catalog, since photoelectric absorption might play an important role. Using the ROSAT catalog we were able

TABLE 3

Sensitivities of Different Hard X-Ray Surveys

\begin{tabular}{|c|c|c|c|c|c|}
\hline Instrument & Reference & $\begin{array}{c}\text { Energy } \\
(\mathrm{keV})\end{array}$ & $\mathrm{S} / \mathrm{N}_{\mathrm{TH}}$ & $\begin{array}{c}\text { Sensitivity } \\
\text { (at } 1 \mathrm{Ms}[\mathrm{mcrab}] \text { ) }\end{array}$ & $\begin{array}{c}\text { Equivalent } 5 \sigma \text { Sensitivity }^{\mathrm{b}} \\
\text { (at } 1 \mathrm{Ms}[\mathrm{mcrab}])\end{array}$ \\
\hline 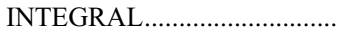 & Bassani et al. (2006) & $20-100$ & 5.0 & 0.8 & 0.8 \\
\hline BAT & Markwardt et al. (2005) & $15-195$ & 5.5 & 1.3 & $1.2^{\mathrm{c}}$ \\
\hline ВAT & This work & $14-170$ & 4.5 & 0.86 & 0.95 \\
\hline
\end{tabular}

${ }^{a}$ The sensitivity is computed by considering the noise-exposure relation provided by the authors (e.g., Fig. 7, right) evaluated at the threshold $\mathrm{S} / \mathrm{N}$ they used to detect sources.

${ }^{\mathrm{b}}$ Sensitivities are computed assuming a threshold $\mathrm{S} / \mathrm{N}$ of $5 \sigma$ for all instruments/surveys.

${ }^{c}$ The sensitivity reported by Markwardt et al. (2005) is referred to the all-sky analysis. Their best $5 \sigma$ sensitivity in high-latitude fields is $\sim 1.0$ mcrab for $1 \mathrm{Ms}$ of exposure. 


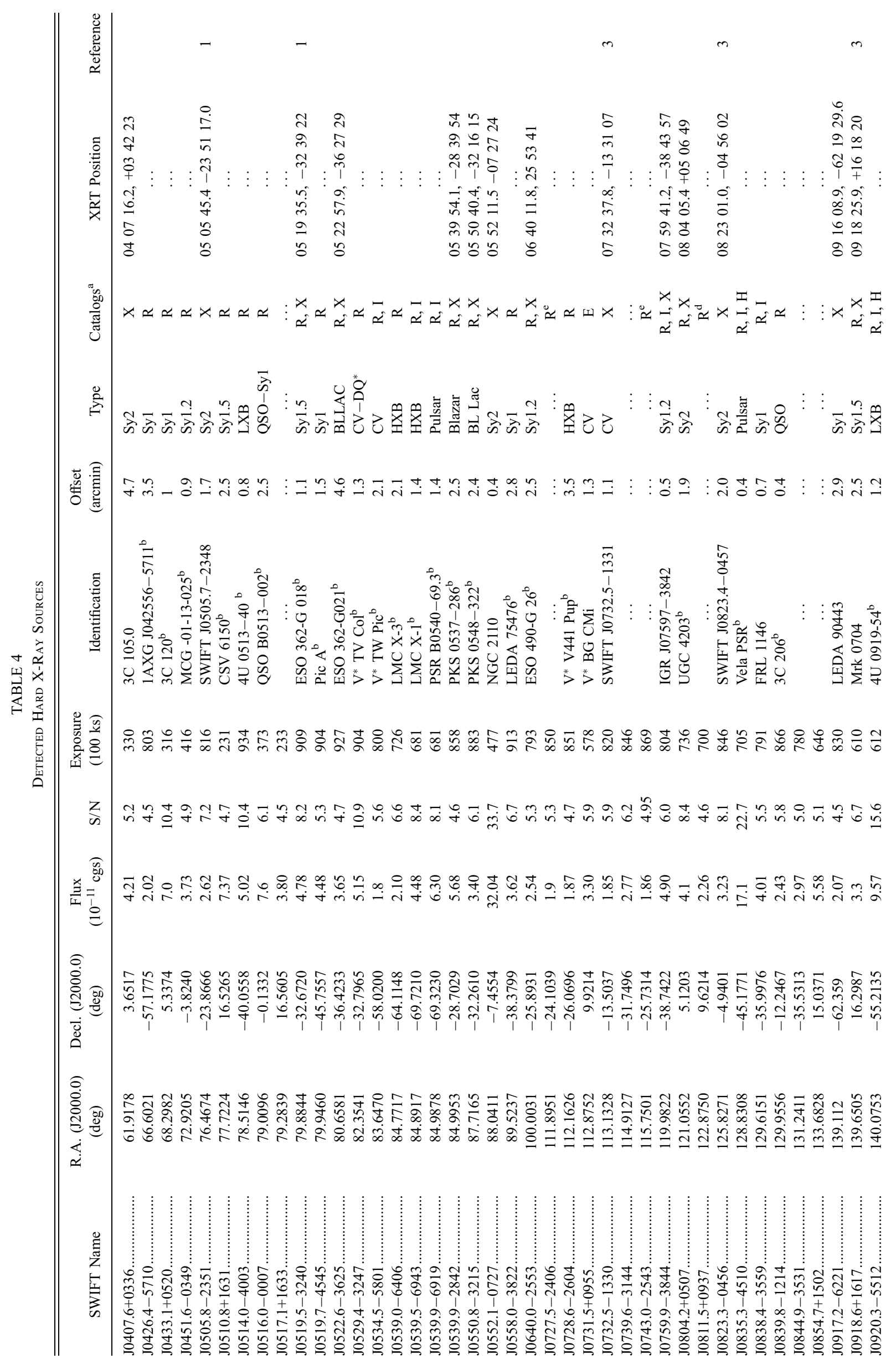




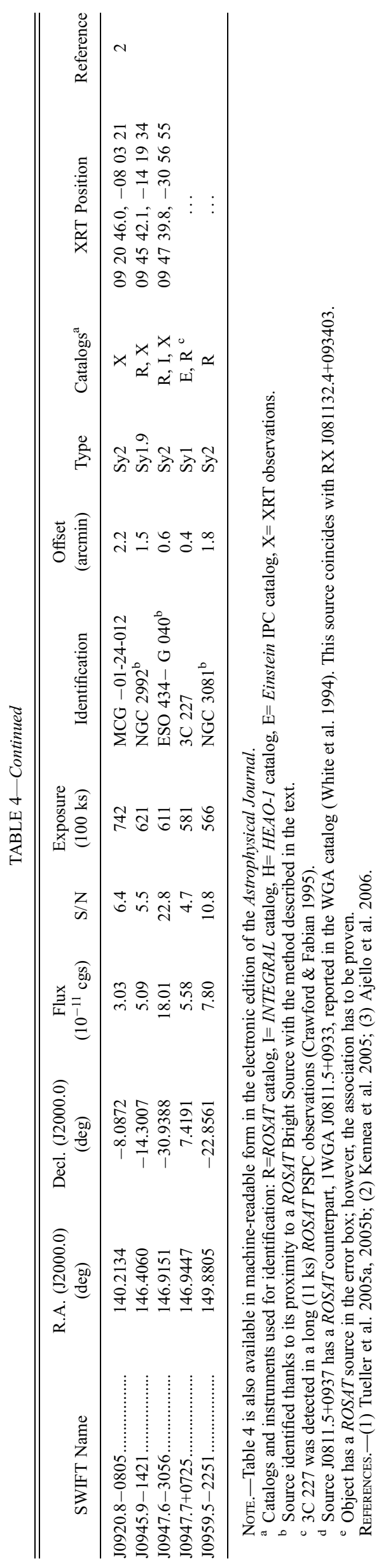




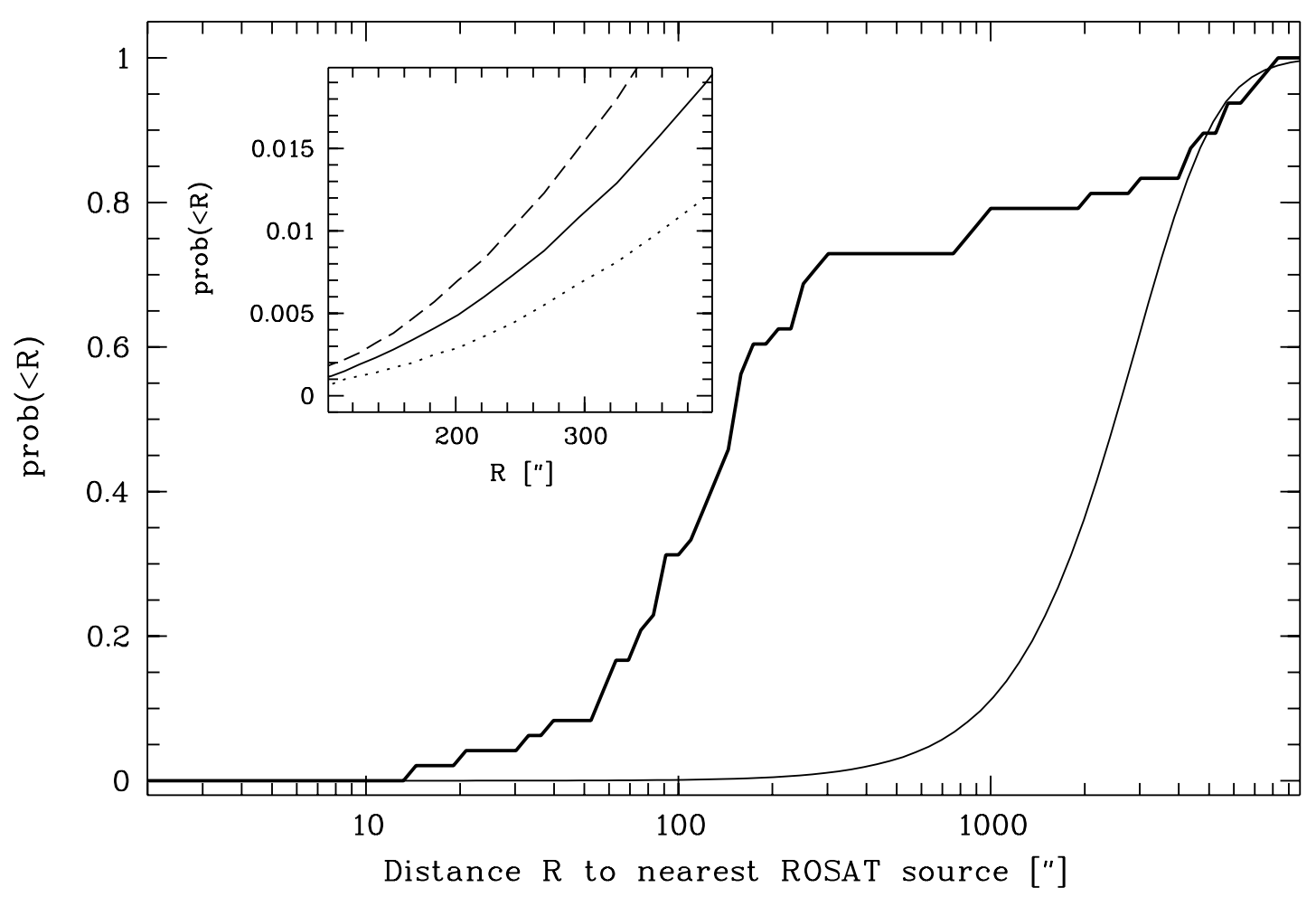

FIG. 8. - Probability of finding at least one ROSAT source within a given radius for our source sample of Table 4. The thick solid line is the probability for the real sample, while the thin line is the expected contribution of chance coincidences. The inset shows a close-up view of the chance coincidence curve for the region of interest $\left(200^{\prime \prime}<R<300^{\prime \prime}\right)$; the dashed line is the chance coincidence distribution for latitudes $-40^{\circ}<b<-20^{\circ}$, while the dotted line is for $0^{\circ}<b<20^{\circ}$.

to identify 30 of our sources. These sources are generally the brightest in our sample, and they were already detected by previous observatories (Macomb \& Gehrels 1999).

Using the same $300^{\prime \prime}$ error radius, we searched for spatial coincidences between our sources and both the HEAO-1 cata$\log$ of high-energy sources (Levine et al. 1984) and the second INTEGRAL IBIS catalog (Bird et al. 2006). We found that two sources had already been detected in hard X-rays by HEAO-1, and seven objects, including also the previous two, by INTEGRAL. All of these seven sources had already been detected at low energy by ROSAT. Two additional sources, $3 \mathrm{C} 227$ and $\mathrm{V}^{*} \mathrm{BG} \mathrm{CMi}$, have an Einstein IPC counterpart (Harris et al. 1990). 3C 227 was also detected during a long (11 ks) ROSAT PSPC observation (Crawford \& Fabian 1995).

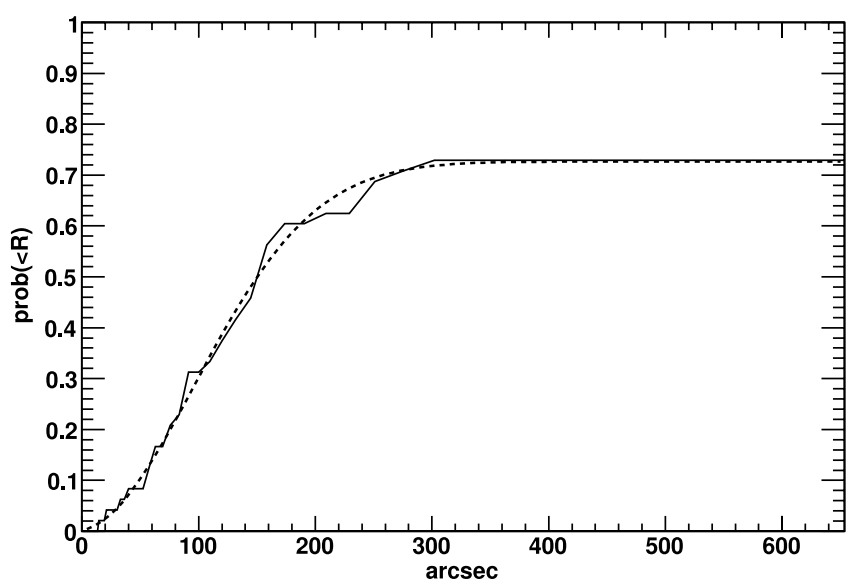

FIG. 9.- Gaussian fit to the curve of Fig. 8. The PSLA at $95 \%$ and $99 \%$ confidence is, respectively, $3.3^{\prime}$ and $5^{\prime}$.
Some of the new sources can be identified using the narrowfield X-ray telescope (XRT) on board Swift. With its 5" position accuracy XRT is able to pinpoint the source counterpart in less than 2 ks. We requested and obtained three follow-up observations of our targets (J0732.5 - 1330, J0823.3 - 0456, and J0918.6+ 1617), and this allowed us to firmly identify the counterpart of those sources (Ajello et al. 2006). Other sources (e.g., J0916.46221, J0519.5-3240, J0505.8-2351, and J0920.8-0805) were observed by XRT as part of the ongoing effort of the BAT all-sky survey (Tueller et al. 2005a, 2005b; Kennea et al. 2005). We also searched the Swift archive for XRT observations covering the fields of our sources.

A total of 17 sources can be firmly identified thanks to XRT. The results of all the identification efforts using X-ray catalogs and XRT are reported in Table 4. Details of all sources identified using XRT are given case-by-case in the next section.

Using the sources with a known X-ray counterpart, we report, in Figure 10, the sources' offsets from their catalog position as a function of significance. A fit to the data shows that the offset varies with $\mathrm{S} / \mathrm{N}$ as

$$
\text { OFFSET }=10.7( \pm 1.9) \times \mathrm{S} / \mathrm{N}^{-0.95( \pm 0.08)} \quad(\operatorname{arcmin}) .
$$

Moreover, from the same plot we expect, for a $4.5 \sigma$ detection, the maximum offset to be $5^{\prime}$; this is in perfect agreement with what is shown in Figure 9. The offset derived for a $10 \sigma$ source from the previous relation and from equation (11) (i.e., the same $10 \sigma$ source is detected in the single $300 \mathrm{~s}$ sky image) is $1.18^{\prime}$ and $1.26^{\prime}$, respectively. The small difference between the per-pointing location accuracy and the accuracy in the summed image is due to the fact that equations (11) and (13) are computed for different ranges of S/N. Indeed, sources detected in the survey image (sum of 2671 shorter observations) span the 4.5-10 range of 


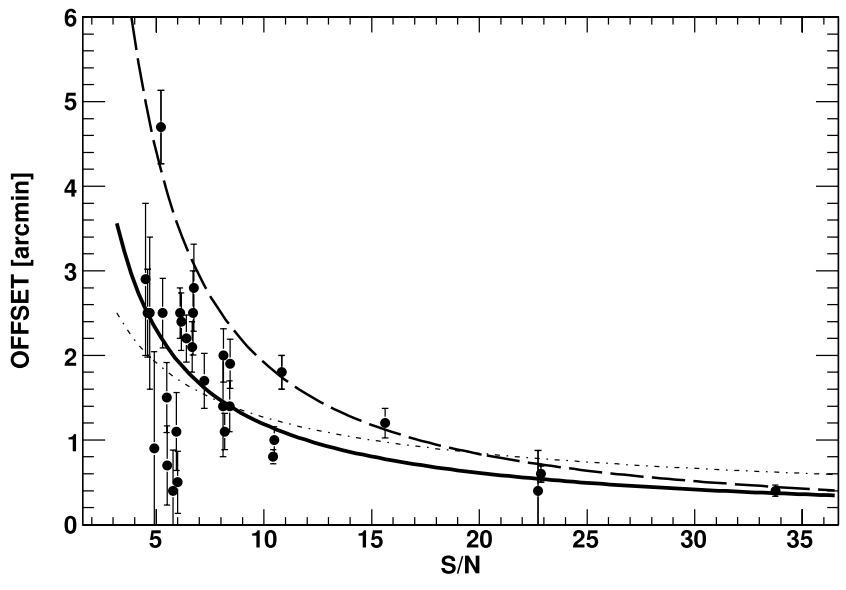

FIG. 10.-Offset of the sources detected in the final survey image as a function of their significance. The solid line is the fit to the data with the function OFFSET $=10.7^{\prime} \times \mathrm{S} / \mathrm{N}^{-0.95}$. The long-dashed line is the fit to $>3 \sigma$ deviations from the previous fit and gives the maximum expected offset for a given significance. For comparison, the short-dashed line shows the best fit (eq. [11]) to the offset-significance relation for sources detected in individual pointings.

significance while most of the sources detected in single pointings have $\mathrm{S} / \mathrm{N}$ greater than $9 \sigma$ (see Fig. 4). Thus, we can affirm that our survey analysis preserves the good location accuracy of BAT.

\subsection{XRT Observations}

SWIFT J0407.6+0336.-XRT observed this source field for $7 \mathrm{ks}$ on 2006 July 11 . The only detected object, at R.A. (J2000.0) = $04^{\mathrm{h}} 07^{\mathrm{m}} 16.2^{\mathrm{s}}$, decl. $(\mathrm{J} 2000.0)=+03^{\circ} 42^{\prime} 24.3^{\prime \prime}$, is coincident with the Seyfert 2 galaxy 3C 105.0 and $4.7^{\prime}$ distant from the BAT position. This is the first time that $3 \mathrm{C} 105.0$ has been detected in X-rays.

SWIFT J0505.8-2351.-XRT observed this source field for $3.2 \mathrm{ks}$ on 2005 August 20, 2005. Only one source is detected within the BAT error box, at R.A. $(\mathrm{J} 2000.0)=05^{\mathrm{h}} 05^{\mathrm{m}} 45.4^{\mathrm{s}}$, decl. $(\mathrm{J} 2000.0)=-23^{\circ} 51^{\prime} 16.8^{\prime \prime}$ coincident with the Seyfert 2 galaxy 2MASX J05054575-2351139. This object was already identified as the BAT counterpart in Tueller et al. (2005b).

SWIFT J0519.5-3240.-A 7 ks XRT observation was performed on 2005 November 26. In the XRT field only two objects are detected. The brighter one, at R.A. (J2000.0) $=05^{\mathrm{h}} 19^{\mathrm{m}} 35.5^{\mathrm{s}}$, decl. $(\mathrm{J} 2000.0)=-32^{\circ} 39^{\prime} 22.4^{\prime \prime}$ is only $1.1^{\prime}$ from the BAT position. The fainter one was detected at R.A. (J2000.0) $=$ $05^{\mathrm{h}} 19^{\mathrm{m}} 25.8^{\mathrm{s}}$, decl. $(\mathrm{J} 2000.0)=-32^{\circ} 42^{\prime} 32.3^{\prime \prime}$, and it is only a $2 \sigma$ detection. The bright source is associated with the nearby Seyfert 1.5 galaxy ESO 362-G018 (also detected by ROSAT as 1RXS J051936.1-323910). ESO 362-G018 was already identified as the BAT counterpart by Tueller et al. (2005b).

SWIFT J0522.6-3625.-XRT observed this field for $899 \mathrm{~s}$ in May 26 2005. Only one source is detected, at R.A. (J2000.0) = $05^{\mathrm{h}} 22^{\mathrm{m}} 57.8^{\mathrm{s}}$, decl. $(\mathrm{J} 2000.0)=-36^{\circ} 27^{\prime} 29.7^{\prime \prime}$ at $4.6^{\prime}$ from the BAT position. The XRT source is coincident with ESO 362-G021 a BL Lac object already detected by ROSAT and XMM-Newton at lower energies and by BeppoSAX in hard X-rays (Donato et al. 2005).

SWIFT J0539.9-2842.-An XRT observation of $14 \mathrm{ks}$ took place on 2005 December 8 . A faint source is detected at R.A. $(\mathrm{J} 2000.0)=05^{\mathrm{h}} 39^{\mathrm{m}} 09.3^{\mathrm{s}}$, decl. $(\mathrm{J} 2000.0)=-28^{\circ} 41^{\prime} 01.5^{\prime \prime}$, coincident with the $z=3.1$ QSO PKS 0537-2843 and 2.5' distant from the BAT position. The QSO was discovered in X-rays by the Einstein Observatory (Zamorani et al. 1981) and then studied by ROSAT, ASCA, and lately by XMM-Newton. The BAT detection in hard X-rays is the first to date.

SWIFT J0550.8-3215.-A 9 ks XRT observation took place on 2005 May 21. A very bright source is detected at R.A. $(\mathrm{J} 2000.0)=05^{\mathrm{h}} 50^{\mathrm{m}} 40.4^{\mathrm{s}}$, decl. $(\mathrm{J} 2000.0)=-32^{\circ} 16^{\prime} 15.5^{\prime \prime}$, $2.4^{\prime}$ distant from the BAT position. The XRT source is associated with a well-known blazar PKS 0548-322, already detected in hard X-ray (see Donato et al. 2005). The blazar is then the BAT counterpart.

SWIFT J0552.1-0727.-During 9 ks of exposure on 2006 April 8, XRT observed a bright source located at R.A. (J2000.0) $=$ $05^{\mathrm{h}} 52^{\mathrm{m}} 11.5^{\mathrm{s}}$, decl. $(\mathrm{J} 2000.0)=-07^{\circ} 27^{\prime} 24.2^{\prime \prime}$. The object is coincident with the well-known Seyfert 2 galaxy NGC 2110, and its position is only $0.4^{\prime}$ away from the BAT detection. The detection of NGC 2110 in the 3-20 keV band by RXTE (Revnivtsev et al. 2004) and the presence of no other source in the XRT field secure the identification of NGC 2110 as the BAT counterpart.

SWIFT J0640.0-2553.-During $2.8 \mathrm{ks}$ of observation on 2006 March 23, XRT observed only one bright source at R.A. $(\mathrm{J} 2000.0)=06^{\mathrm{h}} 40^{\mathrm{m}} 11.8^{\mathrm{s}}$, decl. $(\mathrm{J} 2000.0)=-25^{\circ} 53^{\prime} 41.5^{\prime \prime}$ coincident with the Seyfert 1 galaxy ESO 490-G26 (already detected in soft X-rays by ROSAT as RX J064011-25536). The source position is $2.5^{\prime}$ distant from the BAT detection.

SWIFT J0732.5-1330 (aka SWIFT J0732.5-1331).-XRT observed this field for $4 \mathrm{ks}$ on 2006 April 28. Only one source is detected in the XRT field, at R.A. $(J 2000.0)=07^{\mathrm{h}} 32^{\mathrm{m}} 37.7^{\mathrm{s}}$, decl. $(\mathrm{J} 2000.0)=-13^{\circ} 31^{\prime} 08.6^{\prime \prime}$. This source is coincident with an USNO B1 star J073237.64-133109.0. The source was already identified as the BAT counterpart by Ajello et al. (2006). Follow-up measurements in the optical determined that this source is a new intermediate polar (Wheatley et al. 2006 and references therein).

SWIFT J0759.9-3844.-XRT observed the field of this source for $7 \mathrm{ks}$. Three sources are clearly detected. The brightest of them is located at R.A. $(\mathrm{J} 2000.0)=07^{\mathrm{h}} 59^{\mathrm{m}} 41.2^{\mathrm{s}}$, decl. $(\mathrm{J} 2000.0)=$ $-38^{\circ} 43^{\prime} 57.9^{\prime \prime}$, only $0.5^{\prime}$ away from the BAT position, while the remaining two are more than $10^{\prime}$ distant. The brightest object is coincident with the INTEGRAL source IGR J07597-3842 and with the ROSAT source RX J075942.0-384359. The fact that the only source within $4^{\prime}$ from the BAT position was also detected by INTEGRAL in hard X-rays (den Hartog et al. 2004) makes this source the BAT counterpart. The probable association with an IR point source IRAS $07579-3835$, and a $1.4 \mathrm{GHz}$ radio counterpart (NVSS archive) makes the case for the object having an AGN nature. This source was identified as being a Seyfert 1.2 galaxy during a recent optical spectroscopy follow-up (Masetti et al. 2006b).

SWIFT J0823.3-0456.- Only a single faint source was observed by XRT during $1.2 \mathrm{ks}$ of exposure on 2006 January 6 . The source is located at R.A. $(\mathrm{J} 2000.0)=08^{\mathrm{h}} 23^{\mathrm{m}} 01^{\mathrm{s}}$, decl. $(\mathrm{J} 2000.0)=$ $-04^{\circ} 56^{\prime} 02.5^{\prime \prime}, 2^{\prime}$ away from the BAT detection. The object is coincident with the galaxy FAIRALL 0272 and was already identified as the BAT counterpart by Ajello et al. (2006). An optical follow-up showed that the source is a Seyfert 2 galaxy (Masetti et al. 2006a).

SWIFT J0918.6+1617 (aka SWIFT J0918.5+1618). - This is another source found thanks to our algorithm (Ajello et al. 2006). During an XRT follow-up of $0.6 \mathrm{ks}$, the only detected source is located at R.A. $(\mathrm{J} 2000.0)=09^{\mathrm{h}} 18^{\mathrm{m}} 25.8^{\mathrm{s}}$, decl. $(\mathrm{J} 2000.0)=$ $+16^{\circ} 18^{\prime} 20.8^{\prime \prime}, 2.5^{\prime}$ away from the BAT position and coincident with the galaxy Mrk 704 . Mrk 704 was previously detected in soft X-rays by ROSAT (Schwope et al. 2000). In a recent optical follow-up, the galaxy was found to be a Syl (Masetti et al. 2006a). 
SWIFT J0920.8-0805.-An XRT observation of $8.5 \mathrm{ks}$ took place on 2005 December 10 . Only one source is detected in the entire field. Its position, R.A. (J2000.0) $=09^{\mathrm{h}} 20^{\mathrm{m}} 46.0^{\mathrm{s}}$, decl. $(\mathrm{J} 2000.0)=-08^{\circ} 03^{\prime} 21.8^{\prime \prime}$, is coincident with the Sy2 galaxy MCG -01-24-012, and is 2.2' distant from the BAT position. This object was already identified as the BAT counterpart by Kennea et al. (2005).

SWIFT J0945.9-1421.-An XRT observation of $11 \mathrm{ks}$ took place on 2006 July 8 . The only source detected inside the BAT error box is located at R.A. $(\mathrm{J} 2000.0)=09^{\mathrm{h}} 45^{\mathrm{m}} 42.0^{\mathrm{s}}$, decl. $(\mathrm{J} 2000.0)=-14^{\circ} 19^{\prime} 33.7^{\prime \prime}$. The source is coincident with the Seyfert 1.9 galaxy NGC 2992 and is $1.5^{\prime}$ distant from the BAT detection. The source was already detected in soft X-rays by ROSAT as 1RXS J094541.9-141927.

SWIFT J0947.6-3056.-XRT observed the source field for $10 \mathrm{ks}$ on 2005 December 9. Only one bright source is detected, at R.A. $(\mathrm{J} 2000.0)=09^{\mathrm{h}} 47^{\mathrm{m}} 39.8^{\mathrm{s}}$, decl. $(\mathrm{J} 2000.0)=$ $-30^{\circ} 56^{\prime} 55.4^{\prime \prime}$, coincident with the Seyfert 2 galaxy ESO 434G040 and only $0.4^{\prime}$ distant from the BAT position. The galaxy was also detected in hard X-ray by INTEGRAL (Bird et al. 2006).

\section{CONCLUSIONS}

We have presented an application of the maximum likelihood method as a deconvolution technique used to reconstruct the sky image when dealing with a coded-mask instrument such as BAT. The main difference with other image reconstruction algorithms, such as the standard cross-correlation technique, is that a sky distribution model is forward-folded through the full instrumental response and fitted to the detector plane counts in order to derive the most probable sky image. This is realized in a single step including data from many pointings, and thus no image mosaicking is required. This study was motivated principally by the capabilities of the ML method (1) to fully preserve the statistical information in the data and (2) to reduce the systematic errors connected to mosaicking techniques that other methods cannot avoid. This leads to an improvement in sensitivity over other methods.

Moreover, this study is motivated by the need to use sensitive imaging techniques for the study of the hard X-ray sky. Although deep soft X-ray surveys $(0.5-2.0 \mathrm{keV})$ were able to resolve the majority of the CXB emission into discrete sources (Hasinger et al. 1998), only a minor fraction of the CXB above $8 \mathrm{keV}$ is resolved (Worsley et al. 2005). Furthermore, the bulk of the CXB radiation is emitted around $30 \mathrm{keV}$ (Marshall et al. 1980), and the exact nature of the source population responsible for the background at these energies is unknown because of the low sensitivity of previous hard X-ray telescopes. The BAT coded mask detector, on board the Swift mission, represents a major improvement in sensitivity for imaging of the hard X-ray sky; thus, we tested our ML imaging algorithm on BAT survey data. This study was also complemented by an optical spectroscopy campaign aimed at identifying BAT-discovered extragalactic hard X-ray objects (Rau et al. 2007).

The results presented in the previous sections can be summarized as follows. After screening our data set for bad data, as discussed in $\S 3.1$, the final survey image obtained using the ML method produces a perfectly Gaussian normal noise. We detected 49 hard X-ray sources above the $4.5 \sigma$ detection threshold. Only 12 were previously known as hard X-ray emitters (previously detected by INTEGRAL or HEAO-1). Thirty-seven are new sources detected by BAT due to our image reconstruction method. The correlation of BAT sources with the ROSAT catalog shows the extremely good location accuracy of the BAT instrument which is also preserved by our algorithm. It is also worth noticing that $\sim 30 \%$ of our sources are not correlated with the ROSAT objects; this is most probably due to the presence of photoelectric absorption in some of the new BAT sources. The analysis of the limiting flux as a function of pixel exposure (see Fig. 7) for the reconstructed image sum of all observations, shows that systematic errors do not dominate over statistical ones and that BAT should be able to achieve, in the future, a sensitivity of 0.5 mcrab with $3 \mathrm{Ms}$ of exposure (if systematics remain at this level). The sky coverage shows that $75 \%$ of the survey is covered to flux $\sim 1$ mcrab and all of it to fluxes $>2.0$ mcrab. All of this makes this analysis one of the most sensitive surveys ever performed in the hard X-ray domain.

The optical spectroscopy identification of the new sources and a discussion about the optical properties are left to a separate paper (Rau et al. 2007), while the statistical and spectral X-ray properties will be discussed in Ajello et al. (2008).

M. A. acknowledges N. Gehrels and the BAT team for the warm hospitality and enlightening discussions, A. Yoldas for all his tips and tricks about parallel programming and Python. The anonymous referee is acknowledged for his helpful comments which improved the manuscript. This research has made use of the NASA/IPAC Extragalactic Database (NED), which is operated by the Jet Propulsion Laboratory, California Institute of Technology, of data obtained from the High Energy Astrophysics Science Archive Research Center (HEASARC) provided by NASA's Goddard Space Flight Center, of the SIMBAD Astronomical Database, which is operated by the Centre de Données astronomiques de Strasbourg, of the Sloan Digital Sky Survey (SDSS) managed by the Astrophysical Research Consortium (ARC) for the Participating Institutions, the National Aeronautics and Space Administration, the National Science Foundation, the Department of Energy, the Japanese Monbukagakusho, and the Max Planck Society, and of the ROSAT All Sky Survey maintained by the Max-Planck-Institut für extraterrestrische Physik.
Ajello, M., et al. 2006, ATel, 697, 1

$$
\text { 2008, ApJ, 673, } 96
$$

Barthelmy, S. D., et al. 2005, Space Sci. Rev., 120, 143

Bassani, L., Malizia, A., \& Stephen, J. B. (INTEGRAL AGN Survey Team). 2006, in The Obscured Universe, ed. S. Grebenev, R. A. Sunyaev, \& C. Winkler (ESA SP-622; Noordwijk: ESA), preprint (astro-ph/0610455v1)

Bird, A. J., et al. 2006, ApJ, 636, 765

Comastri, A., Setti, G., Zamorani, G., \& Hasinger, G. 1995, A\&A, 296, 1

Crawford, C. S., \& Fabian, A. C. 1995, MNRAS, 273, 827

den Hartog, P. R., Hermsen, W., Kuiper, L. M., in't Zand, J. J. M., Winkler, C., \& Domingo, A. 2004, ATel, 261, 1

Diehl, R., et al. 1995, A\&A, 298, 445

\section{REFERENCES}

Donato, D., Sambruna, R. M., \& Gliozzi, M. 2005, A\&A, 433, 1163

Fabian, A. C., \& Iwasawa, K. 1999, MNRAS, 303, L34

Gehrels, N., et al. 2004, ApJ, 611, 1005

Giacconi, R., et al. 2002, ApJS, 139, 369

Gilli, R., Salvati, M., \& Hasinger, G. 2001, A\&A, 366, 407

Harris, D. E., et al. 1990, in Imaging X-Ray Astronomy. A Decade of Einstein Observatory Achievements, ed. M. Elvis (Cambridge: Cambridge Univ. Press), 309

Hasinger, G., Burg, R., Giacconi, R., Schmidt, M., Trumper, J., \& Zamorani, G. 1998, A\&A, 329, 482

Kennea, J. A., et al. 2005, ATel, 677, 1

Levine, A. M., et al. 1984, ApJS, 54, 581 
Macomb, D. J., \& Gehrels, N. 1999, ApJS, 120, 335

Markwardt, C. B., Tueller, J., Skinner, G. K., Gehrels, N., Barthelmy, S. D., \& Mushotzky, R. F. 2005, ApJ, 633, L77

Marshall, F. E., Boldt, E. A., Holt, S. S., Miller, R. B., Mushotzky, R. F., Rose, L. A., Rothschild, R. E., \& Serlemitsos, P. J. 1980, ApJ, 235, 4

Masetti, N., Bassani, L., Dean, A. J., Ubertini, P., \& Walter, R. 2006a, ATel, 735,1

Masetti, N., et al. 2006b, A\&A, 459, 21

Rau, A., Greiner, J., Salvato, M., \& Ajello, M. 2007, A\&A, submitted

Revnivtsev, M., Sazonov, S., Jahoda, K., \& Gilfanov, M. 2004, A\&A, 418, 927

Rosati, P., et al. 2002, ApJ, 566, 667

Schwope, A., et al. 2000, Astron. Nachr., 321, 1

Skinner, G. K., Ponman, T. J., Hammersley, A. P., \& Eyles, C. J. 1987, Ap\&SS, 136,337
Stephen, J. B., et al. 2006, A\&A, 445, 869

Strong, A. W., Diehl, R., Halloin, H., Schönfelder, V., Bouchet, L., Mandrou, P., Lebrun, F., \& Terrier, R. 2005, A\&A, 444, 495

Tueller, J., et al. 2005a, ATel, 668, 1

$$
\text { 2005b, ATel, 669, } 1
$$

Voges, W., et al. 1999, A\&A, 349, 389

Wheatley, P. J., Marsh, T. R., \& Clarkson, W. 2006, ATel, 765, 1

White, N. E., Giommi, P., \& Angelini, L. 1994, BAAS, 26, 1372

Wilks, S. S. 1938, Ann. Math. Stat., 9, 60

Worsley, M. A., et al. 2005, MNRAS, 357, 1281

Zamorani, G., et al. 1981, ApJ, 245, 357 